\title{
Estimation of the case fatality rate based on stratification for the COVID-19 outbreak
}

\author{
Byungwon Kim ${ }^{1}$, Seonghong Kim ${ }^{1}$, Woncheol Jang ${ }^{1 *}$, Sungkyu Jung ${ }^{1}$, Johan Lim ${ }^{1}$ \\ 1 Department of Statistics, Seoul National University, Seoul, Korea, Republic of \\ *wcjang@snu.ac.kr
}

\begin{abstract}
This work is motivated by the recent worldwide pandemic of the novel coronavirus disease (COVID-19). When an epidemiological disease is prevalent, estimating the case fatality rate, the proportion of deaths out of the total cases, accurately and quickly is important as the case fatality rate is one of the crucial indicators of the risk of a disease. In this work, we propose an alternative estimator of the case fatality rate that provides more accurate estimate during an outbreak by reducing the downward bias (underestimation) of the naive CFR, the proportion of deaths out of confirmed cases at each time point, which is the most commonly used estimator due to the simplicity. The proposed estimator is designed to achieve the availability of real-time update by using the commonly reported quantities, the numbers of confirmed, cured, deceased cases, in the computation. To enhance the accuracy, the proposed estimator adapts a stratification, that allows the estimator to use information from heterogeneous strata separately. With the COVID-19 cases of China, South Korea and the United States, we numerically show the proposed stratification-based estimator plays a role of providing an early warning about the severity of a epidemiological disease that estimates the final case fatality rate accurately and shows faster convergence to the final case fatality rate.
\end{abstract}

\section{Introduction}

The outbreak of the novel coronavirus disease (COVID-19) epidemic in Wuhan City, Hubei Province, China on December 8, 2019 has sharply grown as a global pandemic. The reported total number of confirmed cases worldwide has exceeded 1.7 million $(1,773,084)$, including 111,652 deaths as of April 13, 2020 [1]. In the early stage of the epidemic, the most cases reported outside Hubei had a history of travel to Wuhan that had effects on the growth of the disease in a few Asian countries, but the impact of recent secondary infections has been wider and faster in the world. To reduce the spread of epidemics, a few policies such as wearing a mask and social distancing are suggested in many countries. See [2] for a research on the effects of social distancing that supports the policy. To implement such policies effectively, we are also interested in risk factors associated with COVID-19.

The case fatality rate (CFR) is one of the important epidemiological measures of disease severity, and is the proportion of deaths among the cases of the disease. As the importance of quick and accurate estimation of the CFR during a disease outbreak is emphasized by many researchers [3 8], the world health organization (WHO) and many countries report the naive CFR daily basis. The naive CFR is the proportion of the cumulative number of deaths out of the cumulative number of confirmed cases at the 
time. It is well known that the naive CFR has a tendency of underestimation due to not counting the quarantined patients at the time, some of which will eventually die.

Many other estimators of the CFR have been proposed to reduce the bias of the naive CFR. For example, 914 proposed to adjust the number of confirmed cases in the denominator by multiplying a factor. [15] proposed to adjust the numerator by adding the expected fatalities of patients in the hospital, the expected fatalities are computed based on the duration of hospitalization.

The reason for using the naive CFR by the WHO and many other countries instead of those proposed alternatives $9 \sqrt{15}$ is that the naive CFR requires minimum information for computation and eventually converges to the final CFR, the proportion of overall deaths out of the total cases when the epidemic is over. During the pandemic of a disease, it is practically infeasible to record all individuals' information accurately and to report it to the public, but most of those proposed estimators require extra personal information. In particular, the estimator of [15] requires daily updates of individuals' statuses from confirmed dates, which is unavailable. For example, in South Korea, since the COVID-19 epidemic started to grow sharply in Daegu on Feb 18, the government has stopped reporting the individual records of infected patients, such as the number of contacts with people, the list of public places visited and the status changes of a patient.

In this paper, we propose an alternative approach for the real-time estimation of CFR. It is designed to reduce the bias of the naive CFR, by accounting for the expected future fatalities as done in [15. To accommodate the real-time update, the proposed model requires only the cumulative numbers of confirmed, deceased and cured patients at the time. While using these simple quantities, to properly adapt characteristics of different groups or partitions in the population, we use a stratification, that estimates the expected number of deaths among the quarantined patients in each stratum separately and then combines those estimated counts to achieve an asymptotic consistency to the final CFR.

The proposed stratification-based model is applied to the cases of China, South Korea, and the United States. For the case of South Korea, the age-group is used for the stratification. For the cases of China and the United States, provinces and states are used for the stratification, respectively. From these examples of application, we show that the proposed model suitably reduces the bias of the naive CFR and converges to the final CFR. The benefit of stratification is greater when each stratum is heterogeneous enough, e.g. the age-group in South Korea, that results in faster convergence to the final CFR than the naive CFR. As a result, by providing an estimate of the case fatality rate that is less biased and converges to the final CFR faster than the naive CFR, the proposed stratification-based model plays a role of providing an early warning of the severity of a disease.

\section{Methods}

We now provide the proposed model for the real-time estimation of the case fatality rate of a disease. Suppose $N(t), C(t), D(t), U(t)$ be the cumulative numbers of the infected, cured, deceased, and quarantined patients at time $t$. The final turnout of the quarantined patients are unknown but they will be eventually cured or deceased. At each $t, N(t)=C(t)+D(t)+U(t)$. We propose an estimator of the final CFR, called a modified naive CFR, at time $t$ as

$$
\widehat{\mathrm{FR}}(t)=\frac{D(t)+E_{u}^{d}(t)}{N(t)},
$$


where $E_{u}^{d}(t)$ denotes the estimated count of deaths among the patients in the hospital $(U(t))$ at time $t$.

The proposed model is designed under an assumption that there exist heterogeneous strata in which the numbers of confirmed, cured, deceased and unknown are available for each stratum. These stratum-wise quantities are used in the calculation of $E_{u}^{d}(t)$. Let $N_{l}(t), C_{l}(t), D_{l}(t), U_{l}(t)$ be the cumulative counts of the infected, cured, deceased and unknown for the stratum $l=1, \ldots, L$. When there is no stratum-wise information, $E_{u}^{d}(t)$ is estimated by letting $L=1$. We now assume, for each stratum $l$, that given $N_{l}(t)$,

$$
\left(C_{l}(t), D_{l}(t), U_{l}(t)\right) \sim \operatorname{Multinomial}\left(N_{l}(t),\left(p_{c}(l, t), p_{d}(l, t), p_{u}(l, t)\right)\right),
$$

and $\left(C_{l}(t), D_{l}(t), U_{l}(t)\right)$ and $\left(C_{\ell}(t), D_{\ell}(t), U_{\ell}(t)\right)$ are independent for $l \neq \ell$. Here, $\left(p_{c}(l, t), p_{d}(l, t), p_{u}(l, t)\right)$ is the time-varying proportion of the cured, deceased and quarantined patients for stratum $l$. We also assume $p_{u}(l, t) \rightarrow 0$ as $t \rightarrow \infty$. Recall, at the end of a disease epidemic, all confirmed patients are determined to be either cured or deceased.

The proposed estimator for the stratum-wise case fatality rate is now defined by

$$
\widehat{\mathrm{FR}}_{l}(t)=\frac{D_{l}(t)+E_{u}^{d}(l, t)}{N_{l}(t)}=\frac{D_{l}(t)}{N_{l}(t)}\left(1+\frac{U_{l}(t)}{N_{l}(t)}\right) .
$$

The estimated count of deaths among the unknown $U(t), E_{u}^{d}(t)$, in $(1)$ is then defined as the sum of $E_{u}^{d}(l, t)$ 's, that leads to the proposed estimator of the case fatality rate (1) as

$$
\widehat{\operatorname{FR}}(t)=\frac{D(t)+\sum_{l=1}^{L} E_{u}^{d}(l, t)}{N(t)}=\frac{D(t)+\sum_{l=1}^{L} U_{l}(t) D_{l}(t) / N_{l}(t)}{N(t)} .
$$

Note that the proposed estimator of the final CFR (3) is a weighted average of the estimator of the stratum-wise CFR (2).

During a disease epidemic, the proposed estimators $(2)$ and (3) prevent underestimation of the case fatality rate by adding the estimated counts of deaths among the quarantined patients to the naive CFR. In addition to the bias correction, the proposed estimators have a consistency, that converges to the final CFR as the outbreak of a disease coming over. To see the convergence, let $p_{d}(l, \infty)$ and $p_{d}(\infty)$ be the final case fatality rates for a stratum $l, l=1, \ldots, L$, and for the population, respectively. Here, we use the infinity symbol, $\infty$, to denote the time-point of the end of epidemic. Though a duration of a disease epidemic is practically finite, but it is unknown during an epidemic, that makes reasonable to use the infinity symbol to indicate an unknown time-point in the future. Recall that the final case fatality rate of a disease is the overall proportion of deaths out of total cases at the end of epidemic, that means there is no more quarantined patients not yet determined to be cured or deceased. This satisfies our assumption, $p_{u}(l, t) \rightarrow 0$ as $t \rightarrow \infty$, that leads to $E_{u}^{d}(l, \infty) \rightarrow 0$ and $E_{u}^{d}(\infty) \rightarrow 0$. Hence, $\widehat{\mathrm{FR}}_{l}(t) \rightarrow p_{d}(l, \infty)$ and $\widehat{\mathrm{FR}}(t) \rightarrow p_{d}(\infty)$ as $t \rightarrow \infty$.

Additional observations for the proposed estimators (2) and (3) are given in the Appendix. In particular, Theorem 1 (ii) and (iii) provide the variances of (2) and (3) which can be used for approximate confidence intervals.

\section{Results}

\section{Province stratified case fatality rates in China}

First of all, we applied the proposed model to the estimation of the final case fatality rate in China. Because the COVID-19 disease originated in Wuhan City and the 
government of China asserted the pandemic of COVID-19 in China was recently almost over, it was meaningful to apply our model to the China case to see the entire flow of the estimated case fatality rates. The data used for the analysis here is available on [16].

The proposed estimator of the final case fatality rate is computed daily basis. Since there were no demographic factors such as age and gender, that the factor-wise information, the numbers of confirmed, deceased and cured patients in each factor group, was available, we used the provinces in China for the stratification. Fig 1 shows the estimated CFR. One can see an weird jump in both the naive CFR and the proposed estimate on Apr 17, that comes from the sudden addition of 1,290 deceased cases in Wuhan announced by the government of Wuhan City, see [17,18 for details. Due to the late report of the missed fatalities, the data published by the government of Wuhan City and followed statistics including the estimates of the CFR have lost trust, but still there is room for interpretation of the flow of our proposed estimates.

When assumed there is no missed fatalities, we see that the proposed estimate computed daily basis has steadily given a warning that the COVID-19 disease is much more danger than what the naive CFR says during the outbreak. In particular, on Jan 31 from which the number of confirmed cases has grown exponentially, our estimate of CFR was $4.22 \%(3.67 \%, 4.77 \%)$ that is close to the final CFR while the naive CFR was only $2.17 \%$. According to Fig 1, the proposed estimate hit the second peak on Feb 25, which is $5.67 \%(5.46 \%, 5.88 \%)$, and then gradually decrease towards $4 \%$. However, if the missed deceased cases were accurately reported from the first, we expect that such a decrease would not happen, that leads to a faster convergence to the current final CFR, $5.55 \%$.

Fig 1. Daily estimates of CFRs in South Korea. Two CFR estimates in China, the daily updated naive CFRs (dotted line) and the proposed province stratified estimates of CFRs (blue solid line) are depicted together. For our proposed estimates, daily basis $95 \%$ confidence intervals (shaded area) are also added.

Fig 2 shows the latest province-wise estimates of CFRs with $95 \%$ confidence intervals for top 10 provinces in the numbers of confirmed cases. Almost confirmed cases (about $81 \%$ ) have been reported in Hubei province whose naive CFR is $6.62 \%$ and our model estimate is $6.63 \%(6.45 \%, 6.82 \%)$ on Apr 19, 2020, while the CFR estimates of other provinces are so much smaller than the estimated CFR .

Fig 2. Latest province-wise CFRs in China. For top 10 provinces in the numbers of confirmed cases, the latest proposed estimate of CFR (blue plus marker) and its $95 \%$ confidence interval (green square brackets) for each province is depicted with the histogram of confirmed cases. The latest naive CFR (red plus marker) calculated for each province separately is also added to show the difference.

\section{Age-group stratified case fatality rates in South Korea}

Next, we applied the proposed model to the estimation of the case fatality rate of the COVID-19 epidemic in South Korea. We used age-groups for stratification. The first confirmed case in South Korea was reported on Jan 20, and the first death was reported on Feb 20, 2020. Since the infected cases were explosively increased in Daegu, once the epicenter of South Korea's outbreak, on Feb 18, the Korea center for disease control and prevention (KCDC) and the ministry of health and welfare (MOHW) stopped publishing individual records of all confirmed patients such as the number of contacts with people, the list of public places visited and patient's status changes. Instead, from Mar 2, MOHW has started to report daily updates of several summary statistics 
including age-group-wise numbers of confirmed and deceased cases. To use age-group information, the results including the below figures are obtained using the data reported by MOHW from Mar 2 to Apr 20, 2020 [19]. The data is also available on [20].

As we did in the case of China, Our estimator of the final CFR is computed daily basis, and as the estimator of the final CFR, it consistently outperforms the naive CFR at each time. To confirm this, we have plotted the proposed estimates of CFR (with \pm 2 standard error) and the naive CFR over time. Fig 3 shows the daily estimated CFRs using the naive CFR, the proposed estimator with and without age-group stratification. From the figure, we first see that there has been no dramatic change in daily estimated CFRs of all three estimates but those CFRs have steadily been increasing. Second, we find that the naive CFR is always outside the $95 \%$ confidence intervals of the proposed estimates (both with and without age-group stratification), showing clear underestimation. Third, from around Mar 25, the rate of increase in the proposed age-group stratified CFR estimate has started to be slower than other two, that can be understood as a signal of fastest convergence to the final CFR. This also shows the stratification can reduce an upward bias which might happen when our proposed model is used without any stratification. Last, on Apr 20, our estimate with age-group stratification was $2.42 \%$ with $95 \%$ confidence interval $(2.13,2.71)$ while the naive CFR was $2.21 \%$. The naive CFR has been inside the confidence interval of the proposed age-group stratified estimate recently, that means the convergence to the final case fatality rate.

Fig 3. Daily estimates of CFRs in South Korea. The daily updated naive CFRs (dotted line), the estimates of CFRs using our model without stratification (red solid line), and the estimates of CFRs using our model with age-group stratification (blue solid line) are depicted together. For our model estimates, both without and with age-group stratification, daily basis $95 \%$ confidence intervals (shaded areas) are also added.

To see a stratification effect, how heterogeneous strata affect on the case fatality rate, we need to check the stratum-wise estimates as well. According to Fig 4 that shows the latest age-group-wise estimates of CFRs with $95 \%$ confidence intervals, the age clearly has a positive relationship with the case fatality rate. See that there has been no deceased patient in those young age groups while almost deceased cases were reported in the elderly age groups. The figure shows that the estimated CFR for the group of $80 \mathrm{~s}$ or over is $23.59 \%(19.79 \%, 27.39 \%)$ and for the group of $70 \mathrm{~s}$ is $11.18 \%$ $(8.69 \%, 13.66 \%)$, both are so much greater than the estimated CFR. Based on the result, we notice that the young age groups, especially the group of 20s, are evidently dropping the CFR by taking large proportions in confirmed cases (largest in 20s) with no deceased cases. In fact, this phenomenon, the group of 20s takes the largest proportion in the number of confirmed cases and lowers the CFR in a significant degree, is highly related with a religious cult, Shincheonji, in South Korea, of which a member played a key role for the outbreak of the COVID-19 infections in Daegu. By attending an assembly of the cult held in Daegu and carelessly getting around many public places with infection confirmed, the famous patient 31 (the 31st confirmed patient in South Korea) made the government unable to identify people who were possibly infected. As a result, the government decided to diagnose all members of the cult, including people without a symptom of the disease. By the decision, the group of 20s became the largest age-group in the confirmed cases, but most of them had no symptom, that affected on lowering the CFR. 
Fig 4. Latest age-group-wise CFRs in South Korea. The latest estimate of CFR (blue plus marker) and its $95 \%$ confidence interval (green square brackets) for each age-group using the proposed age-group stratification model is depicted with the histogram of confirmed cases. The latest naive CFR (red plus marker) calculated for each age-group separately is also added to show the difference.

\section{State stratified case fatality rates in the United States}

The last example is the case of the United States. The recent growth of the COVID-19 pandemic in the United States is much severer than any other country that the numbers of confirmed and deceased cases have exceeded 700 thousand and 37 thousand, respectively, both are now the most cases in the world. The data used for the analysis here is available on 21.

Fig 5 shows the estimated daily case fatality rates in the United States. We find that the flow of CFR estimates in the United States has just passed the first wave, the first sharp increase and decrease of CFRs in the earliest stage of pandemic, that does not give a clue for the final case fatality rate. The figure shows that, after the first wave, both the naive CFR and the proposed estimate with state stratification are still increasing and the gap between these two CFRs is getting wider. Based on the cases of South Korea and China above, we expect that it will take a few more days or weeks for the decrease of daily CFRs and the final convergence. And the state-wise estimates of CFRs, that show significant differences between the proposed estimate and the naive CFR in all states as depicted in Fig 6, also support this expectation.

Fig 5. Daily estimates of CFRs in the United States. Two CFR estimates in the United States, the daily updated naive CFRs (dotted line) and the proposed estimates of CFRs with state stratification (blue solid line) are depicted together. For our model estimates, daily basis $95 \%$ confidence intervals (shaded area) are overlaid.

As the histogram in Fig 6 shows, We know that the number of confirmed cases in the New York state overwhelms other states in the United States, but the estimated CFR, $10.47 \%(10.30 \%, 10.63 \%)$, takes the second place, while the Michigan state is ranked at the first with the estimated CFR, $13.79 \%(13.27 \%, 14.31 \%)$, that is somewhat out of expectation. As analyzed in 22], the reason that the estimated CFR is the highest in the Michigan state might come from the low rate of COVID-19 test, 795 tests taken per 100 thousand people in the Michigan state that is ranked 27th in the United States, but further analysis such as demographic factors stratified estimation of the case fatality rate can be helpful for figuring out the reason.

Fig 6. Latest state-wise CFRs in the United States. For top 10 states in the numbers of confirmed cases, the latest proposed estimate of CFR (blue plus marker) and its $95 \%$ confidence interval (green square brackets) for each state is depicted with the histogram of confirmed cases. The latest naive CFR (red plus marker) calculated for each state separately is also added to show the difference.

\section{Discussion}

In this paper, we proposed a modification of the naive CFR that allowed to estimate the case fatality rate of a disease in real-time basis. The proposed estimator is designed to reduce the downward bias (underestimation) of the naive CFR during an outbreak of a disease, especially in the early stage of an epidemic, and to converge to the final case 
fatality rate. In particular, by adapting a stratification, the proposed estimator is made to combine information from heterogeneous strata appropriately. By the COVID-19 cases of South Korea, China and the United States, we have shown that the proposed estimator outperforms the naive CFR, that provides more accurate estimate during a disease epidemic and converges faster to the final case fatality rate.

Here is an issue that needs to be stated for clear interpretation of the case fatality rate we estimate. As stated in [23], the proposed estimator and the naive CFR do not estimate the true death rate for the COVID-19 disease, which is defined as the proportion of deaths out of all infected cases. Note that all infected cases include the cases with and without symptoms regardless the disease diagnosed or not. This is the problem caused by not counting on people who are infected the disease but not diagnosed in the estimation of the case fatality rate. Under ordinary circumstances, even an infected person does not take a diagnostic test if there is no or little symptom of the disease. In addition, for the current COVID-19 disease, the shortage of the diagnostic kits and expensive costs required for the test and the cure have prevented from detecting all infected patients (even with symptoms) by making people hesitate to take the diagnostic test.

Even in South Korea, a counter example that a large number of infected cases without symptoms of the disease are included in the cumulative number of confirmed cases, it is not guaranteed that all infected cases are detected. Recall that we mentioned about an explosive spread of the COVID-19 disease in Daegu, South Korea, in the Results section. Since then, the government of South Korea has extensively traced and tested people exposed to a danger of infection by meeting infected people or visiting public places where infected people visited. In those tested people, all members of the religious cult, Shincheonji, were included. In addition, a few more solutions, such as exempting the testing fee for the disease diagnosed people and installing drive-thru and walk-thru diagnostic testing places, encouraged people to take the test positively. As a result, in the cumulative number of confirmed cases in South Korea, a large number of cases without a symptom of the COVID-19 disease have been included. Nevertheless, a larger part of asymptomatic cases cannot be detected unless all members of the population are tested.

Accordingly, the number of confirmed cases is counted to be much smaller than the number of infected cases, and as a result, the estimated case fatality rate tends to be greater than the true death rate. Hence, when the proposed estimator is used for the estimation of the case fatality rate as a measure of severity of a disease, this should be stated. Otherwise, to use the proposed estimator as a measure of the true death rate, collaboration with an accurate estimation of the transmissibility of a disease must be required.

\section{Supplementary Materials}

Daily updated estimates of CFRs (1. Age-group stratified CFRs in South Korea, 2. State stratified CFRs in the United States, 3. Province stratified CFRs in China, and 4. Country stratified global CFRs) are posted online, https://sungkyujung.github.io/covid19cfr//. All R functions and example codes used for the calculation of the proposed CFR estimates are also posted.

\section{Author Contributions}

W.J. conceived the study and B.K., S.J. and J.L. participated in the study design including formulation of the proposed estimator. B.K., S.K. and S.J. participated in 
collecting the latest data, analyzing the data, and maintaining online supplementary materials. B.K. drafted the manuscript and all authors have read and agreed to the published version of the manuscript.

\section{Acknowledgments}

W.J. and B.K. were supported by the National Research Foundation of Korea (NRF) grant funded by the Korea government (MSIT) (No. 2017R1A2B2012816). S.J. and B.K. were supported by the National Research Foundation of Korea (NRF) grant funded by the Korea government (MSIT) (No. 2019R1A2C2002256).

\section{Appendix}

\section{Additional observation for the proposed estimator}

For the proposed estimator of the case fatality rate at time $t$, we have the following observation:

Theorem 1. (i) $E\left(\widehat{\mathrm{FR}}_{l}(t)\right)=p_{d}(l, t)+p_{d}(l, t) p_{u}(l, t) \frac{N_{l}(t)-1}{N_{l}(t)}$, and $\widehat{\mathrm{FR}}_{l}(t)$ $(l=1, \ldots, L)$ is asymptotically unbiased as $t \rightarrow \infty$.

(ii) $\operatorname{Var}\left(\widehat{\mathrm{FR}}_{l}(t)\right)=\frac{1}{N_{l}(t)} \eta_{l}(t)+\frac{1}{N_{l}^{2}(t)} \zeta_{l}(t)$, where (dropping $(l, t)$ temporarily)

$$
\eta_{l}(t)=p_{d}\left(1-p_{d}\right)+\frac{N_{l}(t)-1}{N_{l}(t)} p_{d} p_{u}\left\{2-3 p_{d}+p_{u}-4 p_{d} p_{u}\right\}
$$

and

$$
\zeta_{l}(t)=\frac{N_{l}(t)-1}{N_{l}(t)} p_{d} p_{u}\left\{1-2 p_{d}+6 p_{d} p_{u}-2 p_{u}\right\}
$$

(iii) $\widehat{\mathrm{FR}}(t)$ is asymptotically unbiased as $t \rightarrow \infty$, and

$$
\operatorname{Var}(\widehat{\operatorname{FR}}(t))=\sum_{l=1}^{L}\left(\frac{N_{l}(t)}{N(t)}\right)^{2}\left\{\frac{1}{N_{l}(t)} \eta_{l}(t)+\frac{1}{N_{l}^{2}(t)} \zeta_{l}(t)\right\} .
$$

Note that as $t \rightarrow \infty, p_{u}(l, t) \rightarrow 0$ and $\operatorname{Var}\left(\widehat{\operatorname{FR}}_{l}(t)\right) \rightarrow \frac{p_{d}\left(1-p_{d}\right)}{N_{l}(\infty)}$. If $N_{l}(t)$ increases as well, then the second term of $\operatorname{Var}\left(\widehat{\mathrm{FR}}_{l}(t)\right)$ becomes negligible as well.

Proof. Note the following. For $\left(X_{1}, X_{2}, X_{3}\right) \sim \operatorname{Multinomial}\left(n,\left(p_{1}, p_{2}, p_{3}\right)\right)$,

$$
\begin{aligned}
E\left(X_{1}\right)= & n p_{1}, \\
E\left(X_{1} X_{2}\right)= & n(n-1) p_{1} p_{2}, \\
E\left(X_{1}^{2}\right)= & n(n-1) p_{1}^{2}+n p_{1}, \\
E\left(X_{1}^{2} X_{2}\right)= & n(n-1)(n-2) p_{1}^{2} p_{2}+n(n-1) p_{1} p_{2}, \\
E\left(X_{1}^{2} X_{2}^{2}\right)= & n(n-1)(n-2)(n-3) p_{1}^{2} p_{2}^{2} \\
& +n(n-1)(n-2)\left(p_{1}^{2} p_{2}+p_{1} p_{2}^{2}\right)+n(n-1) p_{1} p_{2} .
\end{aligned}
$$

Denote $\left(X_{1}, X_{2}, X_{3}, n\right)=\left(D_{l}(t), U_{l}(t), C_{l}(t), N_{l}(t)\right)$ for ease of presentation. Then (i) $E\left(\widehat{\mathrm{FR}}_{l}(t)\right)=\frac{1}{n} E\left(X_{1}+\frac{X_{1} X_{2}}{n}\right)=\frac{1}{n}\left(n p_{1}+(n-1) p_{1} p_{2}\right)=p_{1}+\frac{n-1}{n} p_{1} p_{2}$. 
(ii) We use

$$
\begin{aligned}
\operatorname{Var}\left(\widehat{\mathrm{FR}}_{l}(t)\right)= & E\left(\widehat{\mathrm{FR}}_{l}^{2}(t)\right)-\left\{E\left(\widehat{\mathrm{FR}}_{l}(t)\right)\right\}^{2}, \\
n^{2} \operatorname{Var}\left(\widehat{\mathrm{FR}}_{l}(t)\right)= & E\left(X_{1}^{2}+\frac{2}{n} X_{1}^{2} X_{2}+\frac{X_{1}^{2} X_{2}^{2}}{n^{2}}\right) \\
& -\left\{n^{2} p_{1}^{2}+2 n(n-1) p_{1}^{2} p_{2}+(n-1)^{2} p_{1}^{2} p_{2}^{2}\right\} .
\end{aligned}
$$

The right hand side is summarized as $\xi_{1}+\xi_{2}$, where

$$
\xi_{1}=n p_{1}\left(1-p_{1}\right)+(n-1) p_{1} p_{2}\left(2-3 p_{1}+p_{2}-4 p_{1} p_{2}\right)
$$

and

$$
\xi_{2}=\frac{n-1}{n} p_{1} p_{2}\left(1-2 p_{1}+6 p_{1} p_{2}-2 p_{2}\right) .
$$

The proof for (iii) is omitted.

\section{References}

1. World Health Organization. Coronavirus disease (COVID-2019) situation reports; Available online: https://www.who.int/emergencies/diseases/ novel-coronavirus-2019/situation-reports/ (accessed on April 20, 2020).

2. Zhang B, Ye T, Heng S, Levy MZ, Small DS. Protocol for an Observational Study on the Effects of Social Distancing on Influenza-Like Illness and COVID-19. arXiv:200402944. 2020+;.

3. Ghani AC, Donnelly CA, Cox DR, Griffin JT, Fraser C, Lam TH, et al. Methods for estimating the case fatality ratio for a novel, emerging infectious disease. American Journal of Epidemiology. 2005;162(5):469-486.

4. Kucharski AJ, Edmunds WJ. Case fatality rate for Ebola virus disease in west Africa. The Lancet. 2014;doi:10.1016/S0140-6736(14)61706-2.

5. Leung G, Lam T, Ho L, Ho S, Chan B, Wong I, et al. The impact of community psychological responses on outbreak control for severe acute respiratory syndrome (SARS) in Hong Kong. Journal of Epidemiology and Community Health. 2003;57(11):857-863.

6. Leung G, Quah S, Ho L, Ho S, Hedley A, Lee H, et al. A tale of two cities: community psychobehavioral surveillance and related impact on outbreak control in Hong Kong and Singapore during the severe acute respiratory syndrome epidemic. Infection Control and Hospital Epidemiology. 2004;25(12):1033-1041.

7. Yu P, Chan J, Fung W. Statistical exploration from SARS. The American Statistician. 2006;60(1):81-91.

8. Philip L H Yu JSKC, Fung WK. Statistical Exploration from SARS. The American Statistician. 2006;60:81 - 91.

9. Yoshikura H. Two parameters characterizing 2009 H1N1 swine influenza epidemic in different countries/regions of the world. Japanese Journal of Infectious Diseases. 2009;62:411-412.

10. Chen Z, Akazawa K, Nakamura T. Estimating the case fatality rate using a constant cure-death hazard ratio. Lifetime Data Analysis. 2009;15(3):316-329. 
medRxiv preprint doi: https://doi.org/10.1101/2020.05.23.20111484; this version posted May 26, 2020. The copyright holder for this preprint (which was not certified by peer review) is the author/funder, who has granted medRxiv a license to display the preprint in perpetuity.

It is made available under a CC-BY-ND 4.0 International license .

11. Yip PS, Lau EH, Lam KF, Huggins RM. A chain multinomial model for estimating the real-time fatality rate of a disease, with an application to severe acute respiratory syndrome. American Journal of Epidemiology.

2005;61(7):700-706.

12. Nishiura H, Klinkenberg D, Roberts M, Heesterbeek JA. Early epidemiological assessment of the virulence of emerging infectious diseases: a case study of an influenza pandemic. PLoS One. 2009;4(8):e6852.

13. Garske T, Legrand J, Donnelly CA, Ward H, Cauchemez S, Fraser C, et al. Assessing the severity of the novel influenza A/H1N1 pandemic. BMJ. 2009;339:b2840.

14. Ejima K, Omori R, Cowling BJ, Aihara K, Nishiura H. The time required to estimate the case fatality ratio of influenza using only the tip of an iceberg: joint estimation of the virulence and the transmission potential. Computational and Mathematical Methods in Medicine. 2012;2012:978901.

15. Lee S, Lim J. Online estimation of the case fatality rate using a run-off triangle data approach: An application to the Korean MERS outbreak in 2015. Statistics in Medicine. 2019;38:2664 - 2679.

16. Johns Hopkins CSSE. The 2019 Novel Coronavirus COVID-19 (2019-nCoV) Data Repository;. Available online: https://github.com/CSSEGISandData/COVID-19 (accessed on April 20, 2020).

17. Xinhuanet. Full text of Wuhan's notification on revising numbers of confirmed COVID-19 cases, deaths;. Available online: http://www.xinhuanet.com/english/2020-04/17/c_138984653.htm

18. World Health Organization. Coronavirus disease 2019 (COVID-19) Situation Report - 88; Available online: https:

//www.who.int/docs/default-source/coronaviruse/situation-reports/ 20200417-sitrep-88-covid-191b6cccd94f8b4f219377bff55719a6ed.pdf? sfvrsn=ebe78315_6.

19. Ministry of Health and Welfare, Republic of Korea. Coronavirus Disease-19 (COVID-19), Republic of Korea;. Available online: http://ncov.mohw.go.kr/en/ (accessed on April 20, 2020).

20. DS4C project. DS4C: Data Science for COVID-19 in South Korea;. Available online: https://www.kaggle.com/kimjihoo/coronavirusdataset (accessed on April 20, 2020).

21. New York Times. An ongoing repository of data on coronavirus cases and deaths in the U.S.;. Available online: https://github.com/nytimes/covid-19-data (accessed on April 20, 2020).

22. Michigan Health Watch. Michigan has highest U.S. coronavirus death rate, ranks low in testing;. Available online: https://www.bridgemi.com/michigan-health-watch/ michigan-has-highest-us-coronavirus-death-rate-ranks-low-testing

23. New York Times. Why we don't know the true death rate for COVID-19; Available online: https://www.nytimes.com/2020/04/17/us/coronavirus-death-rate.html. 
Province-wise Case Fatality Rates in China

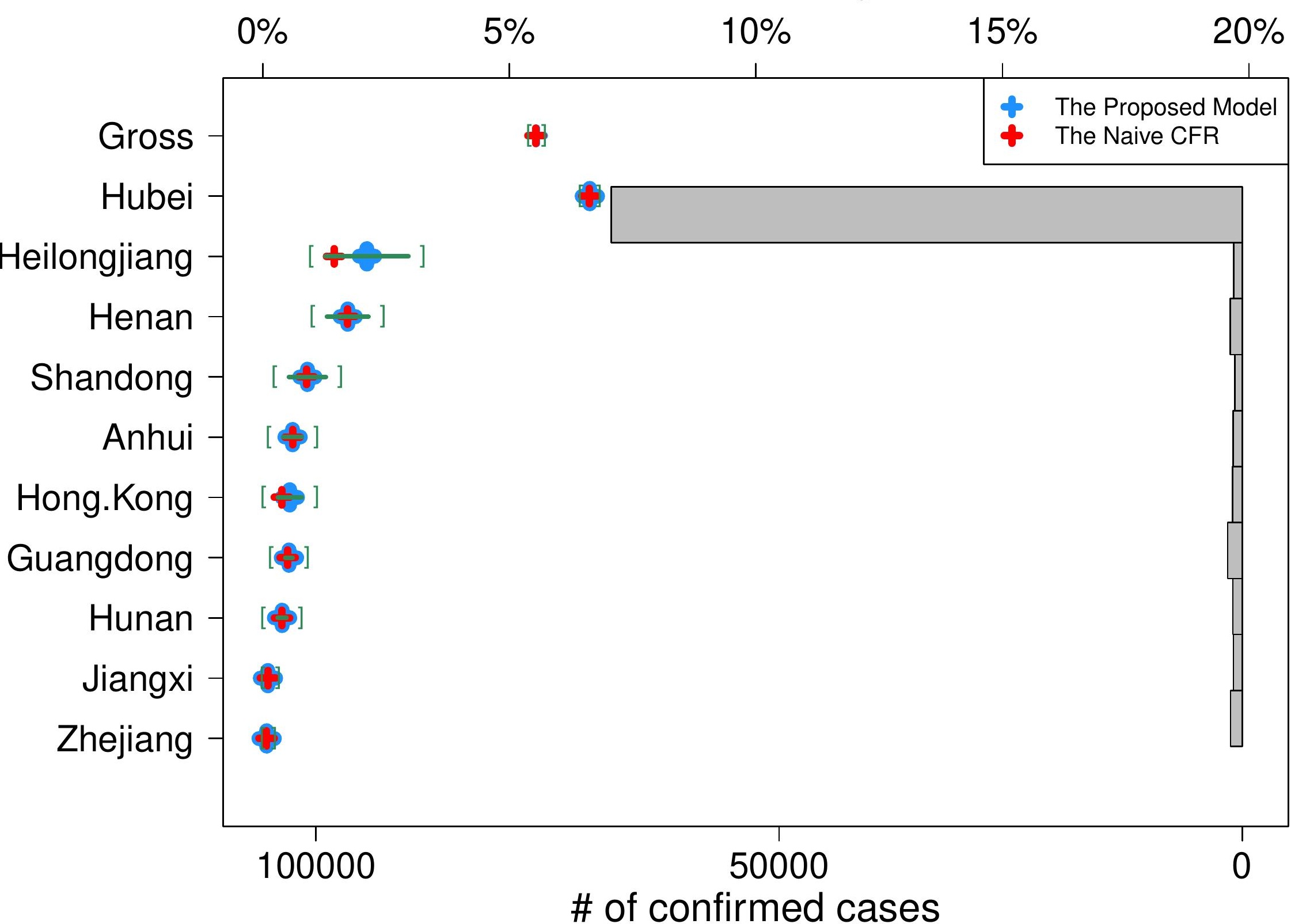




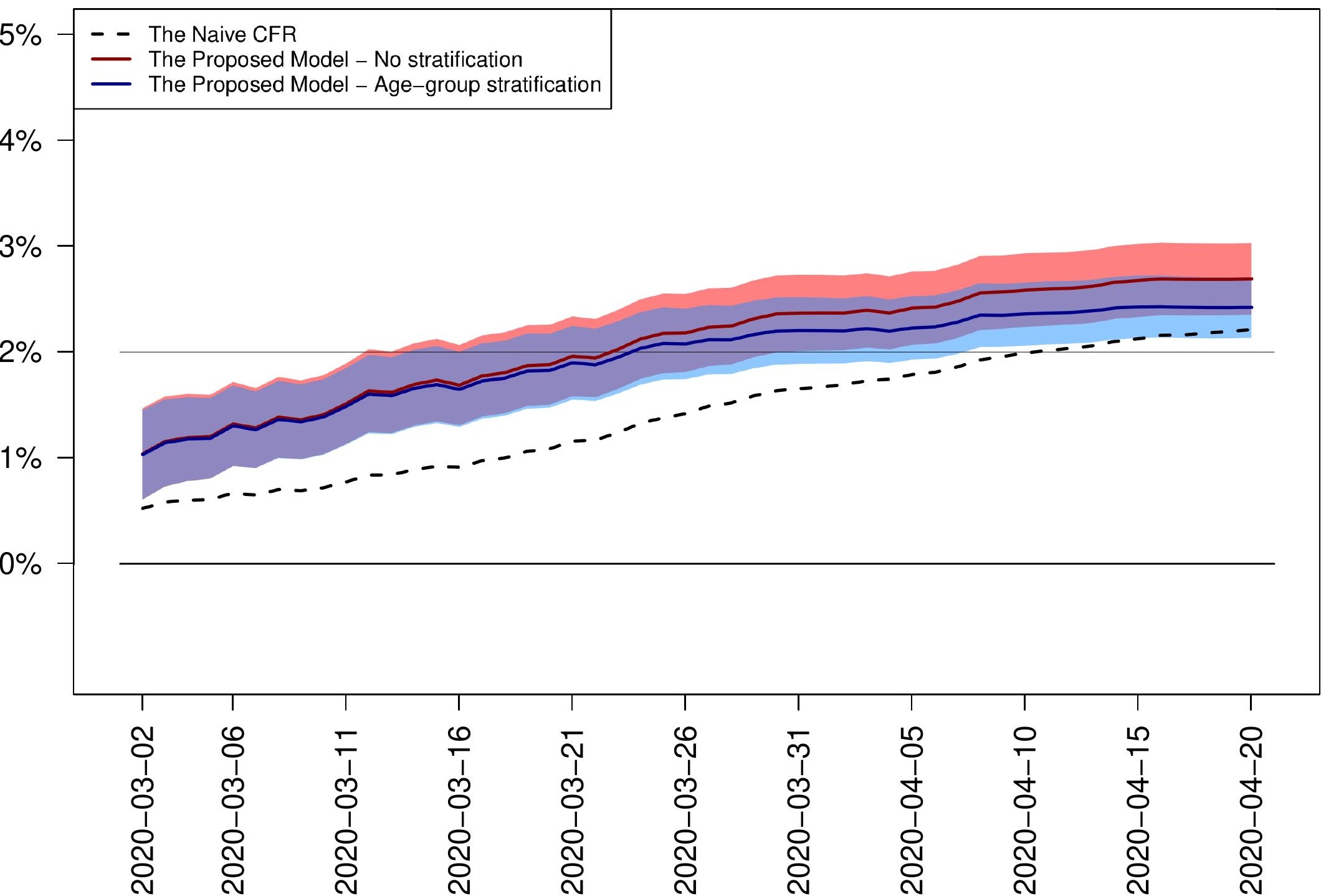


Age-group-wise Case Fatality Rates in South Korea

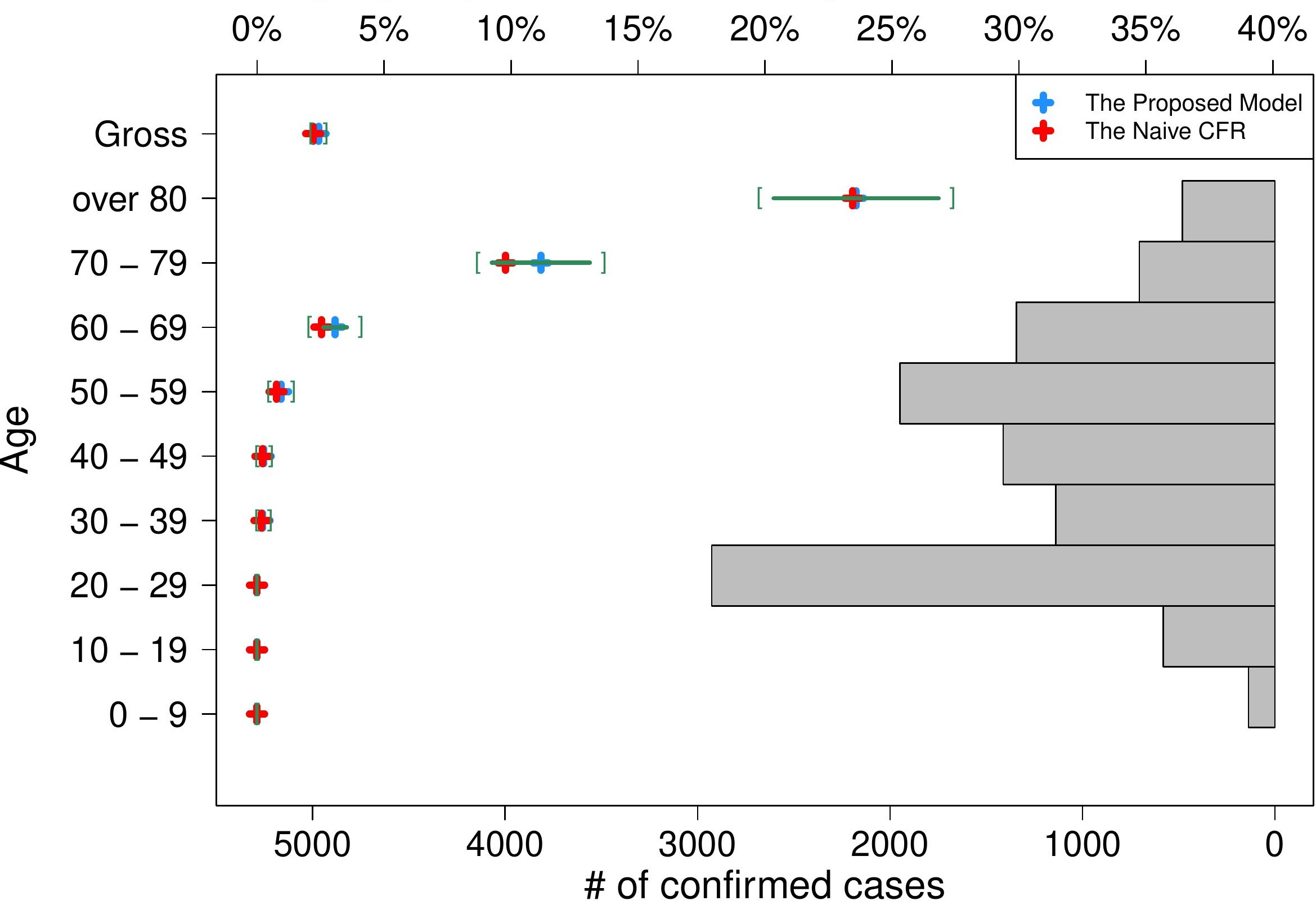




\section{Daily Case Fatality Rate in the United States}




